\title{
Abducens Nerve Disorder, CTCAE
}

National Cancer Institute

\section{Source}

National Cancer Institute. Abducens Nerve Disorder, CT CAE. NCI Thesaurus. Code C143257.

A disorder characterized by dysfunction of the abducens nerve (sixth cranial nerve). 